\title{
PURE POINT SPECTRUM FOR THE MARYLAND MODEL: A CONSTRUCTIVE PROOF
}

\author{
SVETLANA JITOMIRSKAYA AND FAN YANG
}

\begin{abstract}
We develop a constructive method to prove and study pure point spectrum for the Maryland model with Diophantine frequencies.
\end{abstract}

\section{INTRODUCTION}

The Maryland model is a discrete self-adjoint Schrödinger operator on $\ell^{2}(\mathbb{Z})$ of the form

$$
\left(H_{\lambda, \alpha, \theta} u\right)_{n}=u_{n+1}+u_{n-1}+\lambda \tan \pi(\theta+n \alpha) u_{n} .
$$

where $\lambda \in \mathbb{R}$ is called the coupling, $\alpha \in \mathbb{R}$ the frequency, and $\theta \in \mathbb{R}$ is the phase. In this paper we will assume $\alpha \in \mathbb{R} \backslash \mathbb{Q}, \lambda>0$. Let $\Theta \triangleq \frac{1}{2}+\alpha \mathbb{Z}+\mathbb{Z}$. Clearly, for $\theta \in \Theta$ the operator is not well defined. From now on when we say "all $\theta$ ", we will mean " $\theta \notin \Theta$ ".

Maryland model is a linear version of the quantum kicked rotor in the momentum space, originally proposed by Grempel, Fishman and Prange [9. As an exactly solvable example of the family of incommensurate models, it attracts continuing interest in physics, e.g. [3], [6], [8].

For Diophantine frequencies $\alpha$, Maryland model has localization: pure point spectrum with exponentially decaying eigenfunctions, for all $\theta$ [5, 21. In fact, it was recently shown in 14 that $\sigma_{p p}\left(H_{\lambda, \alpha, \theta}\right)$ can be characterized arithmetically, in an exact way, for all parameters. Namely, an index $\delta(\alpha, \theta) \in[-\infty, \infty]$ was introduced in [14] and it was shown that $\sigma_{p p}\left(H_{\lambda, \alpha, \theta}\right)=\left\{E: L_{\lambda}(E) \geq \delta(\alpha, \theta)\right\}$, while $\sigma_{s c}\left(H_{\lambda, \alpha, \theta}\right)=\overline{\left\{E: L_{\lambda}(E)<\delta(\alpha, \theta)\right\}}$ where $L_{\lambda}(E)$ is the Lyapunov exponent, see (2.4) (which for the Maryland model does not depend on $\alpha, \theta)$.

It should be noted that all the proofs of localization so far, including those mentioned above, as well as the original physics paper [9], have been indirect: based on a Cayley transform that reduced the eigenvalue problem to solving certain explicit cohomological equation. In this paper we present a different approach, by proving exponential decay of all polynomially bounded solutions directly. The eigenfunctions of the Maryland model are, as a result of indirect analysis, known exactly, yet the formulas don't make it easy to make conclusions about their behavior, which is quite interesting, with transfer matrices satisfying certain exact renormalization [4. The advantage of our approach is that it provides a completely different and rather promising way to study the solutions, for example, their asymptotics and various other features. For example, the Maryland eigenfunctions are expected, through numerics, to have hierarchical structure driven by the continued fraction expansion of the frequency, and our method has the potential to be developed to study that, as was recently done for the almost Mathieu operator in [15]. Moreover, we expect to be able to also use this method to study some features of solutions/spectral measures/quantum dynamics in the singular continuous regime 1 as was done for the almost Mathieu operator in [17. In general, Maryland model, being exactly solvable, has been a very useful laboratory in the field of quasiperiodic operators, as a source of both general conjectures and counterexamples. The possibility of direct analysis of Maryland eigenfunctions, presented in this manuscript, provides a new very important tool to this laboratory.

\footnotetext{
${ }^{1}$ First studied in physics literature in 20 .
} 
Here we show how the argument works in the simplest, that is Diophantine, case. We will develop a more delicate method and apply it to study the full localization region in the upcoming work [11.

Let $\frac{p_{n}}{q_{n}}$ be the continued fraction approximants of $\alpha \in \mathbb{R} \backslash \mathbb{Q}$. Let

$$
\beta=\beta(\alpha)=\limsup _{n \rightarrow \infty} \frac{\ln q_{n+1}}{q_{n}},
$$

We call $\alpha$ Diophantine if $\beta(\alpha)=0$.

A formal solution $\phi(x)$ of $H \phi=E \phi$ is called a generalized eigenfunction if $\phi$ is a non-trivial solution, and $|\phi(x)| \leq C(1+|x|)$ for some constant $0<C<\infty$.

Our main result is:

Theorem 1.1. For Diophantine $\alpha$ and any $\theta$, any generalized eigenfunction of $H_{\lambda, \alpha, \theta}$ decays exponentially.

Remark 1.1. By Schnol's theorem [2, 10], Theorem 1.1] is equivalent to the statement that $H_{\lambda, \alpha, \theta}$ has pure point spectrum with exponentially decaying eigenfunctions, a known result, as already mentioned. We choose to formulate it the way we do to underscore the new method of proof.

There has been a number of papers lately with constructive proofs of localization with arithmetic conditions for the almost Mathieu and extended Harper's model [13, 1, 19, 15, 16, all dealing with cos potential. Here we show that the method of [12] can also be developed in an even simpler way to treat the Maryland model.

\section{Preliminaries}

Let $\mathbb{T}=\mathbb{R} / \mathbb{Z}$ be the one dimensional torus. For $x \in \mathbb{R}$, let $\|x\|_{\mathbb{T}}=\operatorname{dist}(x, \mathbb{Z})$. Later, we will also sometimes write $\|x\|$ for $\|x\|_{\mathbb{T}}$.

2.1. Cocycles and Lyapunov exponents. For $\alpha \in \mathbb{R}$, and $A: \mathbb{T} \rightarrow M_{2}(\mathbb{C})$ or $A: \mathbb{T} \rightarrow$ $M_{2}(\mathbb{C}) /\{ \pm 1\}$ Borel measurable satisfying

$$
\log |\operatorname{det}(A(\theta))| \in L^{1}(\mathbb{T}),
$$

we call the pair $(\alpha, A)$ a cocycle understood as a linear skew-product on $\mathbb{T} \times \mathbb{C}^{2}\left(\right.$ or $\left.\mathbb{T} \times \mathbb{P} \mathbb{C}^{2}\right)$ defined by

$$
(\alpha, A):(\theta, v) \mapsto(\theta+\alpha, A(\theta) \cdot v)
$$

The Lyapunov exponent of $(\alpha, A)$ is defined by

$$
L(\alpha, A)=\lim _{k \rightarrow \infty} \frac{1}{k} \int_{\mathbb{T}} \ln \|A(\theta+(k-1) \alpha) \cdots A(\theta+\alpha) A(\theta)\| \mathrm{d} \theta .
$$

If we consider the eigenvalue equation of the Maryland model $H_{\lambda, \alpha, \theta} \phi=E \phi$, then any solution can be reconstructed via the following relation

$$
\left(\begin{array}{c}
\phi(k+1) \\
\phi(k)
\end{array}\right)=D(\theta+k \alpha, E)\left(\begin{array}{c}
\phi(k) \\
\phi(k-1)
\end{array}\right)
$$

where

$$
D(\theta, E)=\left(\begin{array}{cc}
E-\lambda \tan \pi \theta & -1 \\
1 & 0
\end{array}\right)
$$

Iterating this process, we will get

$$
\left(\begin{array}{c}
\phi(k) \\
\phi(k-1)
\end{array}\right)=D_{k}(\theta, E)\left(\begin{array}{c}
\phi(0) \\
\phi(-1)
\end{array}\right)
$$


where

$$
\left\{\begin{array}{c}
D_{k}(\theta, E)=D(\theta+(k-1) \alpha, E) \cdots D(\theta+\alpha, E) D(\theta, E) \text { for } k \geq 1, \\
D_{0}(\theta, E)=\mathrm{Id}, \\
D_{k}(\theta, E)=\left(D_{-k}(\theta+k \alpha, E)\right)^{-1} \text { for } k \leq-1 .
\end{array}\right.
$$

The pair $(\alpha, D)$ is the Schrödinger cocycle corresponding to the Maryland model. We will denote the Lyapunov exponent $L(\alpha, D(\cdot, E))$ by $L(E)$. $D_{k}$ is called the $k$-step transfer matrix. It was shown in 9 ] that

$$
e^{L(E)}+e^{-L(E)}=\frac{\sqrt{(2+E)^{2}+\lambda^{2}}+\sqrt{(2-E)^{2}+\lambda^{2}}}{2} .
$$

Note that the cocycle $(\alpha, D)$ is singular because it contains $\tan \pi \theta$. As it is more convenient to work with non-singular cocycles, we introduce

$$
F(\theta, E)=\cos \pi \theta \cdot D(\theta, E)=\left(\begin{array}{cc}
E \cos \pi \theta-\lambda \sin \pi \theta & -\cos \pi \theta \\
\cos \pi \theta & 0
\end{array}\right) .
$$

Note that $F$ is an $M_{2}(\mathbb{R}) /\{ \pm 1\}$ cocycle (that is, defined up to a sign). We will denote its Lyapunov exponent $L(\alpha, F(\cdot, E))$ by $\tilde{L}(E)$. Clearly by (2.2) and the fact that $\int_{\mathbb{T}} \ln |\cos \pi \theta| d \theta=-\ln 2$, we have the following relation between $L(E)$ and $\tilde{L}(E)$.

$$
\tilde{L}(E)=L(E)-\ln 2 \text {. }
$$

The following control of the norm of the transfer matrix of a uniquely ergodic continuous cocycle by the Lyapunov exponent is well known.

Lemma 2.1. (e.g. [7, 18]) Let $(\alpha, M)$ be a continuous cocycle, then for any $\epsilon>0$, for $|k|$ large enough,

$$
\left\|M_{k}(\theta)\right\| \leq e^{|k|(L(\alpha, M)+\epsilon)} \text { for any } \theta \in \mathbb{T} .
$$

Remark 2.1. Considering 1-dimensional continuous cocycles, a corollary of Lemma 2.1 is that if $g$ is a continuous function such that $\ln |g| \in L^{1}(\mathbb{T})$, then for any $\epsilon>0$, and $b-a$ sufficiently large,

$$
\left|\prod_{j=a}^{b} g(\theta+j \alpha)\right| \leq e^{(b-a+1)\left(\int_{\mathbb{T}} \ln |g| \mathrm{d} \theta+\epsilon\right)} .
$$

In particular, we obtain upper bound of $\left|\prod_{j=a}^{b} \cos \pi(\theta+j \alpha)\right|$ as follows:

$$
\left|\prod_{j=a}^{b} \cos \pi(\theta+j \alpha)\right| \leq e^{(b-a+1)(-\ln 2+\epsilon)} .
$$

2.2. A closer look at the transfer matrix. If we consider the Schrödinger cocycle $(\alpha, D(\theta, E))$, it turns out $D_{k}(\theta, E)$ has the following expression

$$
D_{k}(\theta, E)=\left(\begin{array}{cc}
P_{k}(\theta, E) & -P_{k-1}(\theta+\alpha, E) \\
P_{k-1}(\theta, E) & -P_{k-2}(\theta+\alpha, E)
\end{array}\right),
$$

where $P_{k}(\theta, E)=\operatorname{det}\left[\left.\left(E-H_{\theta}\right)\right|_{[0, k-1]}\right]$.

Let $\tilde{P}_{k}(\theta, E): \mathbb{R} / 2 \mathbb{Z} \rightarrow \mathbb{R}$ be defined as $\tilde{P}_{k}(\theta, E)=\prod_{j=0}^{k-1} \cos \pi(\theta+j \alpha) \cdot P_{k}(\theta, E)$. Then clearly

$$
F_{k}(\theta, E)=\left(\begin{array}{cc}
\tilde{P}_{k}(\theta, E) & -\tilde{P}_{k-1}(\theta+\alpha, E) \cos \pi \theta \\
\tilde{P}_{k-1}(\theta, E) \cos \pi(\theta+(k-1) \alpha) & -\tilde{P}_{k-2}(\theta+\alpha, E) \cos \pi \theta \cos \pi(\theta+(k-1) \alpha)
\end{array}\right) .
$$


By the fact that $F$ is continuous and by (2.9) and Lemma 2.1. we have the following upper bound on $\tilde{P}_{k}$.

Lemma 2.2. For any $\epsilon>0$ for $|k|$ large enough,

$$
\left|\tilde{P}_{k}(\theta, E)\right| \leq e^{(\tilde{L}(E)+\epsilon)|k|} \text { for any } \theta \in \mathbb{T} .
$$

2.3. Solution and Green's function. We use $G_{\left[x_{1}, x_{2}\right]}(E)(x, y)$ for the Green's function ( $H$ $E)^{-1}(x, y)$ of the operator $H_{\lambda, \alpha, \theta}$ restricted to the interval $\left[x_{1}, x_{2}\right]$ with zero boundary conditions at $x_{1}-1$ and $x_{2}+1$. We will omit $E$ when it is fixed throughout the argument.

Let $\phi$ be a solution to $H \phi=E \phi$ and let $\left[x_{1}, x_{2}\right]$ be an interval containing $y$. We have

$$
\phi(y)=-G_{\left[x_{1}, x_{2}\right]}\left(x_{1}, y\right) \phi\left(x_{1}-1\right)-G_{\left[x_{1}, x_{2}\right]}\left(x_{2}, y\right) \phi\left(x_{2}+1\right) .
$$

By Cramer's rule, we have the following connection between the determinants $\tilde{P}_{k}$ and Green's function:

$$
\begin{aligned}
\left|G_{\left[x_{1}, x_{2}\right]}\left(x_{1}, y\right)\right| & =\frac{\left|P_{x_{2}-y}(\theta+(y+1) \alpha)\right|}{\left|P_{x_{2}-x_{1}+1}\left(\theta+x_{1} \alpha\right)\right|} \\
& =\frac{\left|\tilde{P}_{x_{2}-y}(\theta+(y+1) \alpha)\right|}{\left|\tilde{P}_{x_{2}-x_{1}+1}\left(\theta+x_{1} \alpha\right)\right|} \prod_{j=x_{1}}^{y}|\cos \pi(\theta+j \alpha)|, \\
\left|G_{\left[x_{1}, x_{2}\right]}\left(x_{2}, y\right)\right| & =\frac{\left|P_{y-x_{1}}\left(\theta+x_{1} \alpha\right)\right|}{\left|P_{x_{2}-x_{1}+1}\left(\theta+x_{1} \alpha\right)\right|} \\
& =\frac{\left|\tilde{P}_{y-x_{1}}\left(\theta+x_{1} \alpha\right)\right|}{\left|\tilde{P}_{x_{2}-x_{1}+1}\left(\theta+x_{1} \alpha\right)\right|} \prod_{j=y}^{x_{2}}|\cos \pi(\theta+j \alpha)| .
\end{aligned}
$$

\subsection{Regular and singular points.}

Definition 2.1. A point $y \in \mathbb{Z}$ will be called $(m, h)$-regular if there exists an interval $\left[x_{1}, x_{2}\right]$, $x_{2}=x_{1}+h-1$, containing $y$, such that,

$$
\left|G_{\left[x_{1}, x_{2}\right]}\left(x_{i}, y\right)\right|<e^{-m\left|y-x_{i}\right|},\left|y-x_{i}\right| \geq \frac{1}{100} h \text { for } i=1,2 .
$$

otherwise, $y$ will be called $(m, h)$-singular.

2.5. Rational approximations. Let $\left\{\frac{p_{n}}{q_{n}}\right\}$ be continued fraction approximants of $\alpha$, then

$$
\begin{aligned}
& \frac{1}{2 q_{n+1}} \leq\left\|q_{n} \alpha\right\| \leq \frac{1}{q_{n+1}}, \\
& \text { and }\|k \alpha\| \geq\left\|q_{n} \alpha\right\| \text { for } 0<|k|<q_{n+1} .
\end{aligned}
$$

If $\alpha$ is Diophantine, then for $n$ large enough, we have

$$
\left\|q_{n} \alpha\right\| \geq e^{-\epsilon q_{n}} .
$$

2.6. Trigonometric product. The following Lemma from [1] gives a useful estimate of products appearing in our analysis.

Lemma 2.3. 1] Let $\alpha \in \mathbb{R} \backslash \mathbb{Q}, \theta \in \mathbb{R}$ and $0 \leq j_{0} \leq q_{n}-1$ be such that

$$
\left|\cos \pi\left(\theta+j_{0} \alpha\right)\right|=\inf _{0 \leq j \leq q_{n}-1}|\cos \pi(\theta+j \alpha)|,
$$


then for some absolute constant $C>0$,

$$
-C \ln q_{n} \leq \sum_{j=0, j \neq j_{0}}^{q_{n}-1} \ln |\cos \pi(\theta+j \alpha)|+\left(q_{n}-1\right) \ln 2 \leq C \ln q_{n}
$$

\section{Key Lemmas}

3.1. Average lower bound of $\tilde{P}_{k}$. We now give the following average lower bound of $\tilde{P}_{k}$ :

Lemma 3.1. For $k$ large enough we have

$$
\frac{1}{k} \int_{\mathbb{T}} \ln \left|\tilde{P}_{k}(\theta)\right| \mathrm{d} \theta=\frac{1}{k} \int_{\mathbb{T}} \ln \left|\tilde{P}_{k}(2 \theta)\right| \mathrm{d} \theta \geq L(E)-\ln 2 .
$$

This lemma will be proved in Section 6 .

3.2. Lagrange interpolation for $\tilde{P}_{k}$. An important observation that makes our analysis possible is

Lemma 3.2. $\tilde{P}_{k}(\theta) / \cos ^{k} \pi \theta$ can be expressed as a polynomial of degree $k$ in $\tan \pi \theta$, namely,

$$
\frac{\tilde{P}_{k}(\theta)}{(\cos \pi \theta)^{k}} \triangleq g_{k}(\tan \pi \theta) \text {. }
$$

Remark 3.1. While $\tilde{P}_{k}(\theta)$ is a function on $\mathbb{R} / 2 \mathbb{Z}, \tilde{P}_{k}(\theta) / \cos ^{k} \pi \theta$ is a function on $\mathbb{R} / \mathbb{Z}$.

Proof. An induction, using that $P_{k}(\theta)=P_{k-1}(\theta)(E-\tan \pi(\theta+(k-1) \alpha))-P_{k-2}(\theta)$.

By the Lagrange interpolation formula, for any set of $k+1$ distinct $\theta_{i}$ 's in $(-1 / 2,1 / 2)$,

$$
g_{k}(\tan \pi \theta)=\sum_{i=0}^{k} g_{k}\left(\tan \pi \theta_{i}\right) \frac{\prod_{l \neq i}\left(\tan \pi \theta-\tan \pi \theta_{l}\right)}{\prod_{l \neq i}\left(\tan \pi \theta_{i}-\tan \pi \theta_{l}\right)} .
$$

Thus we have the following convenient representation

$$
\begin{aligned}
\tilde{P}_{k}(\theta)=(\cos \pi \theta)^{k} g_{k}(\tan \pi \theta) & =\sum_{i=0}^{k} \tilde{P}_{k}\left(\theta_{i}\right) \frac{\prod_{l \neq i} \tan \pi \theta-\tan \pi \theta_{l}}{\prod_{l \neq i} \tan \pi \theta_{i}-\tan \pi \theta_{l}} \cdot \frac{\cos ^{k} \pi \theta}{\cos ^{k} \pi \theta_{i}} \\
& =\sum_{i=0}^{k} \tilde{P}_{k}\left(\theta_{i}\right) \prod_{l \neq i} \frac{\sin \pi\left(\theta-\theta_{l}\right)}{\sin \pi\left(\theta_{i}-\theta_{l}\right)} .
\end{aligned}
$$

\subsection{Uniformity.}

Definition 3.1. We say that the set $\left\{\theta_{1}, \ldots, \theta_{k+1}\right\}$ is $\epsilon$-uniform if

$$
\max _{\theta \in[0,1]} \max _{i=0, \ldots, k} \prod_{l \neq i} \frac{\left|\sin \pi\left(\theta-\theta_{l}\right)\right|}{\left|\sin \pi\left(\theta_{i}-\theta_{l}\right)\right|}<e^{k \epsilon} .
$$

Note that this differs from the definition of uniformity used in [1, 19, 15, 16. For a fixed $k$, choose the largest $q_{n}$ such that $\frac{1}{25} q_{n} \leq|k|$. We will assume $k \geq 0$. We define $I_{1}$ and $I_{2}$ differently in the following two cases:

Case 1. If $\frac{1}{25} q_{n} \leq k<q_{n}$, let $h=2 q_{n}$, and set

$$
\left\{\begin{array}{c}
I_{1}=\left[k-2 q_{n}-\left[\frac{2 q_{n}}{100}\right]+1, k-q_{n}-\left[\frac{2 q_{n}}{100}\right]\right], \\
I_{2}=\left[k-\left[\frac{2 q_{n}}{100}\right]-q_{n}+1, k-\left[\frac{2 q_{n}}{100}\right]\right] .
\end{array}\right.
$$


Case 2. If $q_{n} \leq k<\frac{1}{25} q_{n+1}$, there exists the smallest positive integer $s$ such that

$$
(2 s-1) q_{n} \leq k<(2 s+1) q_{n} .
$$

Let $h=2 s q_{n}$ and set

$$
\left\{\begin{array}{c}
I_{1}=\left[-2 s q_{n}+\left[\frac{2 s q_{n}}{100}\right]+1,-s q_{n}+\left[\frac{2 s q_{n}}{100}\right]\right] \\
I_{2}=\left[k-\left[\frac{2 s q_{n}}{100}\right]-s q_{n}+1, k-\left[\frac{2 s q_{n}}{100}\right]\right]
\end{array}\right.
$$

For both cases, $I_{1} \cup I_{2}$ consists of $h$ points. From now on we fix $0<\epsilon<\frac{L(E)}{600}$. We will show that

Lemma 3.3. For all $k$ sufficiently large, $\{\theta+l \alpha\}_{l \in I_{1} \cup I_{2}}$ is $3 \epsilon$-uniform.

The proof will be given in Section 5 ,

3.4. Upper bound on $\tilde{P}_{h-1}$ on $I_{1}$. We will show that $\tilde{P}_{h-1}$ cannot be large on $I_{1}$, namely,

Lemma 3.4. For $h$ large enough, for any $x_{1} \in I_{1}$, we have $\left|\tilde{P}_{h-1}\left(\theta+x_{1} \alpha\right)\right|<e^{h(\tilde{L}-4 \epsilon)}$.

Proof. We will prove by contradiction. Without loss of generality, assume $\phi(0) \neq 0$. Suppose there exists $x_{1} \in I_{1}$ such that $\left|\tilde{P}_{h-1}\left(\theta+x_{1} \alpha\right)\right| \geq e^{h(\tilde{L}-4 \epsilon)}$. By (2.11) and definition of $I_{1}$, we have

$$
|\phi(0)|=\left|G_{\left[x_{1}, x_{2}\right]}\left(x_{1}, 0\right) \phi\left(x_{1}-1\right)+G_{\left[x_{1}, x_{2}\right]}\left(x_{2}, 0\right) \phi\left(x_{2}+1\right)\right|,
$$

where $x_{1}<0<x_{2}=x_{1}+h-2,\left|x_{i}\right| \geq \frac{h}{100}$.

Using the fact that the numerators of Green's functions can be bounded uniformly by Lemma 2.2. and using (2.6), (2.7), (2.12), (2.13), we can get upper bounds for the following Green's functions:

$$
\begin{aligned}
\left|G_{\left[x_{1}, x_{2}\right]}\left(x_{1}, 0\right)\right| & =\frac{\left|\tilde{P}_{x_{2}}(\theta+\alpha)\right|}{\left|\tilde{P}_{h-1}\left(\theta+x_{1} \alpha\right)\right|} \prod_{j=x_{1}}^{0}|\cos \pi(\theta+j \alpha)|, \\
& \leq 2 e^{-\left|x_{1}\right| L+5 h \epsilon} \\
& \leq 2 e^{-\frac{h}{100}(L-500 \epsilon)} \rightarrow 0 \\
\left|G_{\left[x_{1}, x_{2}\right]}\left(x_{2}, 0\right)\right| & =\frac{\left|\tilde{P}_{-x_{1}}\left(\theta+x_{1} \alpha\right)\right|}{\left|\tilde{P}_{h-1}\left(\theta+x_{1} \alpha\right)\right|} \prod_{j=0}^{x_{2}}|\cos \pi(\theta+j \alpha)| . \\
& \leq 2 e^{-x_{2} L+5 h \epsilon} \\
& \leq 2 e^{-\frac{h}{100}(L-500 \epsilon)} \rightarrow 0
\end{aligned}
$$

For large $h$, this contradicts our assumption $\phi(0) \neq 0$. Therefore, for any $x_{1} \in I_{1}$, we have $\mid \tilde{P}_{h-1}(\theta+$ $\left.x_{1} \alpha\right) \mid<e^{h(\tilde{L}-4 \epsilon)}$.

\subsection{Regularity of $k$.}

Lemma 3.5. For large $|k|$, there exists $x_{1} \in I_{2}$, such that $\left|\tilde{P}_{h-1}\left(\theta+x_{1} \alpha\right)\right| \geq e^{h(\tilde{L}-4 \epsilon)}$

Proof. Consider the Lagrange interpolation of $\tilde{P}_{h-1}(\theta)$ (3.3). By Lemmas 3.3, 3.4, we obtain that it is impossible to have $\left|\tilde{P}_{h-1}\left(\theta+x_{1} \alpha\right)\right|<e^{h(\tilde{L}-4 \epsilon)}$ for all $x_{1} \in I_{1} \cup I_{2}$, as it will contradict Lemma 3.1. Thus we can conclude that there must exist an $x_{1} \in I_{2}$, such that $\left|\tilde{P}_{h-1}\left(\theta+x_{1} \alpha\right)\right| \geq e^{h(\tilde{L}-4 \epsilon)}$.

The existence of such $x_{1}$ leads to the following lemma.

Lemma 3.6. For $|k| \in \mathbb{Z}$ large enough, $k$ is $(L-500 \epsilon, h-1)$-regular. 
Remark 3.2. By our choice of $h$, we have $\frac{2}{3}|k| \leq h \leq 50|k|$

Proof. Since $x_{2}=x_{1}+h-2$, again the numerators of both expressions in (2.12) and (2.13) can be controlled using (2.7) and Lemma 2.2. that is

$$
\begin{aligned}
& \left|\tilde{P}_{x_{2}-k}(\theta+(k+1) \alpha)\right| \leq e^{(\tilde{L}(E)+\epsilon)\left(x_{2}-k+1\right)}, \\
& \left|\tilde{P}_{k-x_{1}}\left(\theta+x_{1} \alpha\right)\right| \leq e^{(\tilde{L}(E)+\epsilon)\left(k-x_{1}+1\right)}, \\
& \prod_{j=x_{1}}^{k}|\cos \pi(\theta+j \alpha)| \leq e^{(-\ln 2+\epsilon)\left(k-x_{1}+1\right)}, \\
& \prod_{j=k}^{x_{2}}|\cos \pi(\theta+j \alpha)| \leq e^{(-\ln 2+\epsilon)\left(x_{2}-k+1\right)} .
\end{aligned}
$$

The regularity is then immediate from the definition and Lemma 3.5 .

\section{Proof of Theorem 1.1}

Applying Lemma 3.6. we obtain that for large $|k|$ there exists an interval $\left[x_{1}, x_{2}\right], x_{2}=x_{1}+h-2$, containing $k$, such that

$$
\left|G_{\left[x_{1}, x_{2}\right]}\left(x_{i}, k\right)\right| \leq e^{-(L-500 \epsilon)\left|k-x_{i}\right|},\left|k-x_{i}\right| \geq \frac{h}{100} \text { for } i=1,2 .
$$

Thus by (2.11),

$$
\begin{aligned}
|\phi(k)| & \leq\left|G_{\left[x_{1}, x_{2}\right]}\left(x_{1}, k\right) \phi\left(x_{1}-1\right)\right|+\left|G_{\left[x_{1}, x_{2}\right]}\left(x_{2}, k\right) \phi\left(x_{2}+1\right)\right| \\
& \leq e^{-(L-500 \epsilon)\left|k-x_{1}\right|}\left|\phi\left(x_{1}-1\right)\right|+e^{-(L-500 \epsilon)\left|k-x_{2}\right|}\left|\phi\left(x_{2}+1\right)\right| \\
& \leq e^{-\frac{h}{100}(L-500 \epsilon)}\left(1+C\left|x_{1}-1\right|+1+C\left|x_{2}+1\right|\right) \\
& <e^{-|k|\left(\frac{L}{150}-4 \epsilon\right)}
\end{aligned}
$$

\section{Proof of Lemma 3.3}

For any $i \in \mathbb{Z}$, let $\theta_{i}:=\theta+i \alpha$.

\subsection{Case 1:}

$$
\frac{1}{25} q_{n} \leq k<q_{n}, h=2 q_{n}
$$

We divide the $2 q_{n}$ points into two intervals: $T_{1}, T_{2}$, each interval containing $q_{n}$ points. Fix any $i$. Let $\left|\sin \pi\left(\theta_{i}-\theta_{l_{j}}\right)\right|$ be the minimal one of $\left|\sin \pi\left(\theta_{i}-\theta_{l}\right)\right|$ in each $T_{j}, j=1,2$. Without loss of generality, assume $i \in T_{1}$. Then $l_{1}=i$ and for any $x \in \mathbb{T}$ we have

$$
\begin{aligned}
& \prod_{l \neq i} \frac{\left|\sin \pi\left(x-\theta_{l}\right)\right|}{\left|\sin \pi\left(\theta_{i}-\theta_{l}\right)\right|} \\
= & \exp \left\{\sum_{l \neq i} \ln \left|\sin \pi\left(x-\theta_{l}\right)\right|-\sum_{l \neq i} \ln \left|\sin \pi\left(\theta_{i}-\theta_{l}\right)\right|\right\}
\end{aligned}
$$


We estimate the two parts separately. First, using Lemma 2.3 .

$$
\begin{aligned}
& \sum_{l \neq i} \ln \left|\sin \pi\left(x-\theta_{l}\right)\right| \\
= & \sum_{j=1}^{2} \sum_{l \in T_{j}, l \neq i} \ln \left|\sin \pi\left(x-\theta_{l}\right)\right| \\
< & 2 q_{n}(-\ln 2+\epsilon) .
\end{aligned}
$$

The maximum distance between $i$ and $l_{2}$ is $2 q_{n}$. However, it may exceed $q_{n+1}$. In this case, $q_{n+1}$ must be equal to $q_{n}+q_{n-1}$. Thus we have the following estimates, using Lemma 2.3 and(2.17):

$$
\begin{aligned}
& \sum_{l \neq i} \ln \left|\sin \pi\left(\theta_{i}-\theta_{l}\right)\right| \\
= & \sum_{l \in T_{1}, l \neq i} \ln \left|\sin \pi\left(\theta_{i}-\theta_{l}\right)\right|+\sum_{l \in T_{2}} \ln \left|\sin \pi\left(\theta_{i}-\theta_{l}\right)\right| \\
\geq & 2\left(-C \ln q_{n}-\left(q_{n}-1\right) \ln 2\right)+\ln \left\|q_{n+1} \alpha\right\| \\
\geq & 2 q_{n}(-\ln 2-\epsilon)-\epsilon q_{n+1} \\
\geq & 2 q_{n}(-\ln 2-2 \epsilon) .
\end{aligned}
$$

Besides, if $2 q_{n}$ does not exceed $q_{n+1}$, the estimate will be:

$$
\begin{aligned}
& \sum_{l \neq i} \ln \left|\sin \pi\left(\theta_{i}-\theta_{l}\right)\right| \\
\geq & 2\left(-C \ln q_{n}-\left(q_{n}-1\right) \ln 2\right)+\ln \left\|q_{n} \alpha\right\| \\
\geq & 2 q_{n}(-\ln 2-\epsilon)-\epsilon q_{n} \\
> & 2 q_{n}(-\ln 2-2 \epsilon) .
\end{aligned}
$$

Therefore we get

$$
\prod_{l \neq i} \frac{\left|\sin \pi\left(x-\theta_{l}\right)\right|}{\left|\sin \pi\left(\theta_{i}-\theta_{l}\right)\right|} \leq e^{3 h \epsilon}
$$

\subsection{Case 2:}

$$
q_{n} \leq k<\frac{1}{25} q_{n+1}, h=2 s q_{n}
$$

We divide the $2 s q_{n}$ points into $2 s$ intervals: $T_{1}, \cdots, T_{2 s}$, each containing $q_{n}$ points. Fix any $i$. Let $\left|\sin \pi\left(\theta_{i}-\theta_{l_{j}}\right)\right|$ be the minimal one of $\left|\sin \pi\left(\theta_{i}-\theta_{l}\right)\right|$ in $T_{j}, j=1, \cdots, 2 s$. Without loss of generality, assume $i \in T_{j_{0}}, 1 \leq j_{0} \leq s$.

We again estimate the two parts in (5.1) separately. Using (2.7) and Lemma 2.3.

$$
\begin{aligned}
& \sum_{l \neq i} \ln \left|\sin \pi\left(x-\theta_{l}\right)\right| \\
\leq & \left(C \ln q_{n}-\left(q_{n}-1\right) \ln 2\right)+(2 s-1)\left(\epsilon-q_{n} \ln 2\right) \\
\leq & 2 s q_{n}(-\ln 2+\epsilon) .
\end{aligned}
$$


And

$$
\begin{aligned}
& \sum_{l \neq i} \ln \left|\sin \pi\left(\theta_{i}-\theta_{l}\right)\right| \\
\geq & 2 s\left(-C \ln q_{n}-\left(q_{n}-1\right) \ln 2\right)+\sum_{j=1, j \neq j_{0}}^{s} \ln \left|\sin \pi\left(\theta_{i}-\theta_{l_{j}}\right)\right|+\sum_{j=s+1}^{2 s} \ln \left|\sin \pi\left(\theta_{i}-\theta_{l_{j}}\right)\right| \\
\geq & 2 s q_{n}(-\ln 2-\epsilon)+I+I I,
\end{aligned}
$$

where we set $I=\sum_{j=1, j \neq j_{0}}^{s} \ln \left\|\left(i-l_{j}\right) \alpha\right\|$ and $I I=\sum_{j=s+1}^{2 s} \ln \left\|\left(i-l_{j}\right) \alpha\right\|$. Note that in the upper bound it is enough to use Lemma 2.3 in each term, leading immediately to a bound by $2 s q_{n}(-\ln 2+\epsilon)$ but we present the estimate the way we do, for clarity.

For $I$, the maximum distance between $i$ and $l_{j}$ is $s q_{n}$, which is clearly smaller than $k$, thus than $q_{n+1}$. Therefore by (2.17), for large $|k|$,

$$
I=\sum_{j=1, j \neq j_{0}}^{s} \ln \left\|\left(i-l_{j}\right) \alpha\right\| \geq(s-1) \ln \left\|q_{n} \alpha\right\| \geq-s q_{n} \epsilon .
$$

For $I I$, the maximum distance between $i$ and $l_{j}$ is $\left(k+2 s q_{n}\right)$, which is smaller than $\frac{3}{25} q_{n+1}$, thus than $q_{n+1}$. Therefore we also have

$$
I I=\sum_{j=s+1}^{2 s} \ln \left\|\left(i-l_{j}\right) \alpha\right\| \geq s \ln \left\|q_{n} \alpha\right\| \geq-s q_{n} \epsilon .
$$

Combining all the estimates above together, we get

$$
\prod_{l \neq i} \frac{\left|\sin \pi\left(x-\theta_{l}\right)\right|}{\left|\sin \pi\left(\theta_{i}-\theta_{l}\right)\right|} \leq e^{3 \epsilon h}
$$

as desired.

\section{Proof of Lemma 3.1}

Proof. We have

$$
\tilde{P}_{k}(2 \theta)=\operatorname{det}\left[\begin{array}{ccccc}
t_{0} & c_{0} & & & \\
c_{1} & t_{1} & c_{1} & & \\
& c_{2} & \cdots & & \\
& & & \cdots & c_{k-2} \\
& & & c_{k-1} & t_{k-1}
\end{array}\right]_{k \times k}
$$

where $t_{j} \triangleq E \cos 2 \pi\left(\theta+\frac{j}{2} \alpha\right)-\lambda \sin 2 \pi\left(\theta+\frac{j}{2} \alpha\right)$ and $c_{j} \triangleq-\cos 2 \pi\left(\theta+\frac{j}{2} \alpha\right)$. Denote $z=e^{2 \pi i \theta}$. Then

$$
\left\{\begin{array}{l}
\tilde{t}_{j}(z) \triangleq e^{\pi i j \alpha} z \cdot t_{j}(z)=\frac{E+i \lambda}{2} e^{2 i \pi j \alpha} z^{2}+\frac{E-i \lambda}{2} \\
\tilde{c}_{j}(z) \triangleq e^{\pi i j \alpha} z \cdot c_{j}(z)=\quad-\frac{1}{2} e^{2 i \pi j \alpha} z^{2}-\frac{1}{2} .
\end{array}\right.
$$


Since $|z|=1$, we have

$$
\left|\tilde{P}_{k}(2 \theta)\right|=\left|f_{k}(z)\right|=\left|\operatorname{det}\left[\begin{array}{ccccc}
\tilde{t}_{0}(z) & \tilde{c}_{0}(z) & & & \\
\tilde{c}_{1}(z) & \tilde{t}_{1}(z) & \tilde{c}_{1}(z) & & \\
& \tilde{c}_{2}(z) & \ldots & & \\
& & & \ldots & \tilde{c}_{k-2}(z) \\
& & & \tilde{c}_{k-1}(z) & \tilde{t}_{k-1}(z)
\end{array}\right]_{k \times k}\right|
$$

Clearly, $\ln \left|f_{k}(z)\right|$ is a subharmonic function, therefore

$$
\begin{aligned}
& \frac{1}{k} \int_{\mathbb{T}} \ln \left|\tilde{P}_{k}(2 \theta)\right| \mathrm{d} \theta=\frac{1}{k} \int_{\mathbb{T}} \ln \left|f\left(e^{2 \pi i \theta}\right)\right| \mathrm{d} \theta \geq \frac{1}{k} \ln \left|f_{k}(0)\right| . \\
& f_{k}(0)=\operatorname{det}\left[\begin{array}{ccccc}
(E-i \lambda) / 2 & -1 / 2 & & & \\
-1 / 2 & (E-i \lambda) / 2 & -1 / 2 & & \\
& -1 / 2 & \cdots & \ldots & -1 / 2 \\
& & & \cdots / 2 & (E-i \lambda) / 2
\end{array}\right]_{k \times k} \\
& =\frac{1}{(-2)^{k}} \operatorname{det}\left[\begin{array}{ccccc}
i \lambda-E & 1 & & \\
1 & i \lambda-E & 1 & & \\
& 1 & \cdots & & \\
& & & \cdots & 1 \\
& & & 1 & i \lambda-E
\end{array}\right]_{k \times k} \triangleq \frac{1}{(-2)^{k}} d_{k} .
\end{aligned}
$$

Obviously $d_{k}=(i \lambda-E) d_{k-1}-d_{k-2}$. Thus

$$
\left|d_{k}\right| \sim C\left|x_{2}\right|^{k-1} \text { as } k \rightarrow \infty,
$$

where $\left|x_{1}\right|<1<\left|x_{2}\right|$ are solutions of the characteristic equation

$$
x^{2}-(i \lambda-E) x+1=0 .
$$

Therefore by (6.2), (6.3) and (6.4), we have2

$$
\lim _{k \rightarrow \infty} \frac{1}{k} \int_{0}^{1} \ln \left|\tilde{P}_{k}(\theta)\right| \mathrm{d} \theta \geq \ln \left|x_{2}\right|-\ln 2 .
$$

Clearly, $x_{2}$ is also the larger (in absolute value) eigenvalue of the following constant matrix

$$
D_{\infty}=\left(\begin{array}{cc}
i \lambda-E & -1 \\
1 & 0
\end{array}\right)
$$

and by (2.2), $\ln \left|x_{2}\right|$ equals to $L\left(\alpha, D_{\infty}\right)$. Then by a simple argument in the proof of Theorem 5.3 of [14] (based on the continuity of the Lyapunov exponent and quantization of acceleration), we have $\ln \left|x_{2}\right|=L\left(\alpha, D_{\infty}\right)=L(E)$. Thus by (6.5) we have

$$
\lim _{k \rightarrow \infty} \frac{1}{k} \int_{0}^{1} \ln \left|\tilde{P}_{k}(\theta)\right| \mathrm{d} \theta \geq L(E)-\ln 2 .
$$

\footnotetext{
${ }^{2}$ From this point on the proof can also be easily finished by a direct computation of $x_{2}$ and using the explicit expression for $L(E)$ in 9 .
} 


\section{ACKNOWLEDGEMENT}

This research was partially supported by NSF DMS-1401204. F.Y. would like to thank Rui Han for many useful discussions, and Qi Zhou for some suggestions.

\section{REFERENCES}

1. A. Avila, S. Jitomirskaya. The ten Martini problem. Annals of Mathematics, 170 (2009): 303-342.

2. Y. M. Berezansky. Expansions in eigenfunctions of selfadjoint operators. American Mathematical Society, (1968), Providence, RI.

3. M. Berry. Incommensurability in an exactly-soluble quantal and classical model for a kicked rotator. Physica D, 10 (1984): 369378.

4. A. Fedotov and F. Sandomirskiy. An exact renormalization formula for the Maryland model. Communications in Mathematical Physics 334.2 (2015): 1083-1099.

5. A. L. Figotin, L.A.Pastur. An exactly solvable model of a multidimensional incommensurate structure. Communications in Mathematical Physics 95.4 (1984): 401-425.

6. S. Fishman. Anderson localization and quantum chaos maps. Scholarpedia, (2010) 5(8):9816.

7. A. Furman, On the multiplicative ergodic theorem for uniquely ergodic systems Annales de l'Institut Henri Poincare (B) Probability and Statistics. No longer published by Elsevier, 33(6): 797-815 (1997).

8. S. Ganeshan, K. Kechedzhi, and S. Das Sarma. Critical integer quantum hall topology and the integrable maryland model as a topological quantum critical point. Physical Review B 90.4 (2014): 041405.

9. D. Grempel, S. Fishman, and R. Prange. Localization in an incommensurate potential: An exactly solvable model. Physical Review Letters, 49.11(1982): 833.

10. R. Han. Shnol's theorem and the spectrum of long range operators. Proceedings of the AMS, 147(7) (2019), 2887-2897.

11. R. Han, S. Jitomirskaya and F. Yang. Universal hierarchical structure of eigenfunctions in the Maryland model. (in preparation)

12. S. Jitomirskaya, Metal-insulator transition for the almost Mathieu operator. Annals of Mathematics 150.3 (1999): 1159-1175.

13. S. Jitomirskaya, D. A. Koslover and M. S. Schulteis. Localization for a family of one-dimensional quasiperiodic operators of magnetic origin. Annales Henri Poincare 6.1 (2005): 103-124.

14. S. Jitomirskaya, W. Liu. Arithmetic spectral transitions for the Maryland model. CPAM, (2017), (70)no.6, 10251051.

15. S. Jitomirskaya, W. Liu. Universal hierarchical structure of quasiperiodic eigenfunctions. Annals of Mathematics (2) 187 (2018), no. 3, 721-776

16. S. Jitomirskaya, W. Liu. Universal reflective-hierarchical structure of quasiperiodic eigenfunctions and sharp spectral transitions in phase, (2018) arXiv preprint arXiv:1802.00781

17. S. Jitomirskaya, W. Liu, and S. Tcheremchantzev. Wavepacket spreading and fractal spectral dimension of quasiperiodic operators with singular continuous spectrum. (working title)

18. S. Jitomirskaya and R. Mavi, Dynamical bounds for quasiperiodic Schrödinger operators with rough potentials. Int. Math. Res. Not. 1, 96-120 (2017).

19. W. Liu, X. Yuan. Anderson Localization for the almost Mathieu operator in exponential regime. Journal of Spectral Theory, 5.1 (2015):89-112.

20. R. Prange, D. Grempel, and S. Fishman. Wave functions at a mobility edge: an example of a singular continuous spectrum. Physical Review B 28.12 (1983): 7370.

21. B. Simon. Almost periodic Schrödinger operators. IV. The Maryland model. Annals of Physics 159.1 (1985): $157-183$.

Svetlana Jitomirskaya, szhitomi@math.uci.edu

Department of Mathematics, University of California, Irvine, California, USA

Fan Yang, ffyangmath@gmail.com

Department of Mathematics, University of California, Irvine, California, USA

Current address: School of Math, Georgia Institute of Technology, Atlanta, Georgia, USA 